\title{
Experiencia de las parteras en la identificación de mujeres maltratadas durante el embarazo
}

Rosario Valdez-Santiago, MA, (1) Luz A renas-Monreal, MSP, D ra en Antropol,(1) Isabel Hernández-Tezoquipa, Ph D Soc.(1)

Valdez-Santiago R,Arenas-Monreal L, Hernández-Tezoquipa I. Experiencia de las parteras en la identificación de mujeres maltratadas durante el embarazo. Salud Publica Mex 2004;46:56-63. El texto completo en inglés de este artículo está disponible en: http://www.insp.mx/salud/index.html

\section{Resumen}

Objetivo. Explorar las experiencias de las parteras en la detección de mujeres maltratadas durante el embarazo, además de conocer los tipos de violencia que identifican con mayor facilidad. Material y métodos. Estudio cualitativo en donde se entrevistó, entre enero y septiembre de 2001, a 12 parteras que forman parte del grupo de parteras del Instituto $\mathrm{N}$ acional de Antropología e H istoria de Morelos, México. Se hizo una selección de aquellas que se ubicaban en Cuernavaca y sus alrededores. Resultados Se reporta: a) el proceso de identificación de violencia realizado por las parteras en la atención prenatal, el cual se basa en un patrón de conductas y actitudes de las mujeres maltratadas tales como descuido en su persona, timidez y, sobre todo, una falta de control en las decisiones sobre el cuidado a su salud, lo cual permite a las parteras hacer preguntas directas so bre violencia; b) las violencias que identifican las parteras con mayor facilidad son la física y la emocional. La violencia sexual fue más difícil para su identificación de manera directa. Conclusiones. Incluir a las parteras dentro de los planes y programas para atender a la violencia intrafamiliar, debido a que su práctica permite una respuesta de apoyo directo a las mujeres maltratadas que lo solicitan. Se propone desarrollar estrategias de capacitación especializada para esta población que atiende a un sector importante de mujeres embarazadas en México. El texto completo en inglés de este artículo está disponible en:http://www.insp.mx/salud/index.html

Palabras claves: parteras; embarazo; violencia doméstica; México

\author{
Valdez-Santiago R,Arenas-Monreal L, Hernández-Tezoquipa I. \\ The midwives' experiences in the identification \\ of battered women in pregnancy. \\ Salud Publica Mex 2004;46:56-63. \\ The English version of this paper \\ is available at: http://www.insp.mx/salud/index.html
}

\begin{abstract}
A bstract
Objective.To explore the experiences of midwives in the identification of battered women during pregnancy and to describe the types of violence they identify most easily. Material and Methods. A qualitative study was conducted between January and September 2001 among twelve midwives from a group of midwives ascribed to the Instituto $\mathrm{N}$ acional de Antropología e Historia (IN AH, N ational Institute of Anthropology and History), in Morelos, Mexico. Participants were selected from those living in Cuernavaca City and surrounding areas. Results. Study findings show that: a) the identification of battered pregnant women in prenatal care should be triggered by a pattern of behaviors and attitudes of battered women, such as being unkempt, shyness, and above all, poor control over decisions on their health care; b) physical and emotional violence were the most frequently types of violence identified by midwives during prenatal care visits. Sexual violence was more difficult to identify. Conclusions It is necessary to incorporate midwives in the national plans and programs to prevent domestic violence. Midwives' practices provide opportunities to care for battered women. Specialized training strategies must be directed to midwives caring for pregnant women in Mexico.The English version of this paper is available at: http://www.insp.mx/salud/index.html
\end{abstract}

Key words: midwives; pregnancy; do mestic violence; Mexico

Este trabajo recibió apoyo económico del programa de financiamiento del Programa Interdisciplinario de Estudios de la Mujer (PIEM) del Colegio de México (2000).

(1) Instituto Nacional de Salud Pública, Cuernavaca, Morelos, México.

Fecha de recibido: 23 de abril de 2003 - Fecha de aprobado: 20 de agosto de 2003

Solicitud de sobretiros: Rosario Valdez Santiago. Instituto N acional de Salud Pública. A venida Universidad 655, 20 piso cubículo 238 colonia Santa María A huacatitlán, 62508 Cuernavaca, Morelos, México.

Correo electrónico: rvaldez@insp.mx 
H oy en día, en el estado de Morelos, al igual que en otras zonas del país, las parteras juegan un papel importante en la atención de los nacimientos. Algunas de las razones para que esto suceda son: a) históricas: en Mesoamérica las parteras o comadronas jugaban un papel central en la atención y cuidados de las mujeres y los bebés en el momento del embarazo, parto y el puerperio. Este predominio continúa durante la mayor parte de la época colonial. Fue hasta la segunda mitad del siglo XVIII cuando la hegemonía de las parteras comenzó a perder terreno, entre otras razones porque se reglamentó su ejercicio y se subordinó su labor a los médicos titulados; ${ }^{1-3}$ b) la existencia de un sistema mixto de atención a la salud, donde interactúan la medicina doméstica, la tradicional, la institucional y la medicina privada; ${ }^{4} \mathrm{c}$ ) la inaccesibilidad a los servicios de salud por parte de un importante sector de la población, por razones estructurales propias al sistema de salud institucional como por cuestiones culturales, tanto de la población que consulta como por la del equipo de salud. Es más, en algunas regiones aisladas y marginadas del país la medicina tradicional y doméstica son la única opción para la atención a la salud para un sector amplio de la población. ${ }^{5}$

Pese a su subordinación y marginación en la atención de los partos, en la actualidad sus servicios son demandados por miles de mujeres, tanto en zonas urbanas como rurales. Diversos estudios ${ }^{6-8}$ han documentado la importancia del papel de las parteras como agentes de salud comunitaria, en cuyo campo de acción interviene también la atención a otros aspectos de la salud reproductiva, como la planificación familiar y los cuidados materno- infantiles.

En estudios hechos en Morelos hace más de una década, aproximadamente $50 \%$ de las mujeres embarazadas del área rural eran atendidas por parteras. ${ }^{9}$ Estos datos son consistentes con un estudio anterior realizado en el ámbito nacional, ${ }^{10}$ en el que a partir de datos provenientes de localidades menores de 2500 habitantes se calculó que las parteras atendían 63\% de los nacimientos. En 1987, la Encuesta Nacional Fertilidad y Salud (Enfes) reportó que las parteras tradicionales atienden $44.5 \%$ de los nacimientos en comunidades de menos de 2500 habitantes y $23.7 \%$ en comunidades mayores de 20000 habitantes. ${ }^{11}$

\section{La violencia durante el embarazo y su impacto en la salud de la mujer y del bebé}

La exploración del vínculo entre violencia y salud reproductiva ha sido escasa en nuestro medio, a pesar de las diferentes formas en que se expresa: alta prevalencia de maltrato durante el embarazo, maternidad forzada -producto de la violación dentro y fuera del matrimonio, maltrato frecuente a embarazadas adolescentes, contagio de infecciones de transmisión sexual-; todo lo anterior tiene un impacto directo en el aumento de la morbilidad-mortalidad materna.

La violencia doméstica durante el embarazo se comenzó a estudiar a finales de la década de los setenta y principios de la de los ochenta. Los estudios correspondientes se llevaron a cabo principalmente en países desarrollados como Estados Unidos de América y Canadá; algunos informes ${ }^{12}$ muestran una variación en la prevalencia que fluctúa entre $9 \%$ y $20.1 \%$ según el tipo y la edad de la población estudiada. Se ha documentado ampliamente que la violencia en contra de la mujer embarazada tiene efectos adversos en la salud de la madre y del bebé; por ejemplo, una agresión física o sexual que implique traumas abdominales puede provocar abruptio placentae -el cual, según el tiempo de gestación, podría llevar a la pérdida del embarazo-; la precipitación del parto o el nacimiento de un bebé de bajo peso. ${ }^{13,14}$ Otras consecuencias posibles de los traumas abdominales incluyen fracturas fetales, la ruptura del útero, hígado o bazo de la madre, fracturas de la pelvis y hemorragias antes del parto. ${ }^{15}$

Es importante considerar que los efectos del maltrato durante el embarazo no sólo producen lesiones físicas; impactan, además, la salud mental de las mujeres. Esto afecta también a las embarazadas, quienes terminan padeciendo diferentes malestares emocionales como depresión, fatiga, apatía, insomnio y tristeza. ${ }^{16}$

En México, el estudio de la asociación entre violencia y el embarazo ha sido reciente. En el primer estudio se exploraron las características de la violencia y el impacto del maltrato durante el embarazo, y se identificó 33.5\% de prevalencia entre las mujeres que acudieron al servicio de ginecobstetricia del Hospital Civil de Cuernavaca; la mayoría fueron maltratadas por sus parejas. Además, se demostró la asociación entre violencia durante el embarazo y efectos adversos en el parto, entre los que destacó el bajo peso al nacer, ya que los bebés de las mujeres maltratadas tuvieron una diferencia de peso de hasta 560 gramos, en comparación con los de las que no sufrieron maltrato. ${ }^{17}$

En otro estudio posterior se identificó una prevalencia de $24.6 \%$ de violencia durante el embarazo, y $24.4 \%$ en el año previo, entre mujeres que acudieron al control prenatal en los Servicios de Salud de Morelos (SSM) y el Instituto Mexicano del Seguro Social (IMSS), tanto en la ciudad de Cuernavaca como en la de Cuautla. Sin embargo, cuando se observaron los datos de 
prevalencia de las usuarias de los SSM, éstas tuvieron una prevalencia de violencia de $33 \%$ en comparación con $15 \%$ de las usuarias del IMSS.*

En México, al igual que en otros países de América Latina, la identificación del maltrato en mujeres embarazadas durante la atención prenatal no es un hecho frecuente. ${ }^{18-20}$ Esto se debe, entre otras razones, a que aún no se reconoce a la violencia como un problema de salud pública que afecta también a las mujeres embarazadas y a sus hijos, por lo que no se ha incorporado a la historia clínica perinatal para así facilitar su identificación oportuna. Por otro lado, a pesar de la publicación de la Norma Oficial Mexicana NOM-190-SSA1-1999, "Prestación de Servicios de Salud. Criterios para la atención Médica de la Violencia Familiar", ${ }^{21}$ hasta el momento son pocas las entidades que han emprendido actividades dirigidas a su implantación. Esta situación de indefinición administrativa aunada a la falta de recursos económicos para llevar a cabo las actividades mínimas de difusión de la norma, tienen como resultado que el personal de salud desconozca la existencia de este importante instrumento.

Con base en lo anterior, el objetivo del presente estudio fue explorar las experiencias de las parteras en la detección de mujeres maltratadas durante el embarazo, además de conocer los tipos de violencia que identifican con mayor facilidad.

\section{Material y métodos}

A través de metodología cualitativa se llevaron a cabo 12 entrevistas semiestructuradas a parteras seleccionadas entre un grupo organizado por el Instituto Nacional de Antropología e Historia (INAH), delegación Morelos, el cual ha auspiciado varias investigaciones sobre las prácticas y recursos de las parteras en este estado. ${ }^{22,23}$ La selección se hizo a partir de un directorio que aglutina a 184 parteras de toda la entidad. Este directorio cuenta con la siguiente información: nombre, edad, dirección, teléfono (en algunos casos) y localidad de residencia de cada una de las integrantes. ${ }^{\ddagger}$

\footnotetext{
* Castro R, Valdez R, Arenas L, Ruiz A. Violencia contra mujeres embarazadas en Morelos (México): un estudio sociológico sobre prevalencia y severidad. 2001. En prensa.

‡ Las localidades incluidas en el directorio son Xochitepec, Santa María, Coatlán del Río, Temixco, Jiutepec, Cuernavaca, Ahuatepec, Tetela del Volcán, Zapata, Axochiapan, Mazatepec, Amacuzac, Acatlipa, Tlayacapan, Yautepec, Cuautla, Totolapa, Tepalcingo, Anenecuilco, Zacatepec, Tlaltizapán, Tlaquiltenango, Jojutla, Jonacatepec, Puente de Ixtla, Tlalnepantla, Huitzilac, Tepoztlán, Ocotepec y Ocuituco.
}

Se hizo una selección de aquellas que se ubicaban en Cuernavaca y sus alrededores. En todos los casos se hizo una visita al domicilio o llamada telefónica a las parteras para explicarles los objetivos del estudio, y si aceptaban participar, se concertaba una cita posterior. Las entrevistas se realizaron en sus casas o en las instalaciones del INAH, según lo dispusiera la informante.

El proceso de recolección de la información se realizó de enero a septiembre de 2001. En las entrevistas se buscó explorar las experiencias de las parteras en la identificación de mujeres maltratadas durante el embarazo. El tiempo promedio de la entrevista fue de $1 \mathrm{~h}$ con 15 min. Además de las entrevistas se aplicó una cédula de información sociodemográfica y se incluyó un diario de campo. Se elaboró una guía de entrevista, la cual se fue adaptando durante el proceso de investigación. Se decidió suspender las entrevistas por haber alcanzado el punto de saturación teórica.

La recolección y análisis de la información incorpó las siguientes estrategias:

Todas las entrevistas fueron grabadas e inmediatamente transcritas para ser revisadas.

Se procesó cada entrevista para poder realizar el análisis con el apoyo del programa de cómputo Ethnograph 4.0.

Se realizó una codificación fina de cada una de las entrevistas; para ello se elaboró un listado de categorías analíticas.

\section{Resultados}

Se desarrollaron las siguientes categorías analíticas: a) proceso de identificación de las mujeres maltratadas en la atención; b) tipos de violencia que identifican las parteras; c) características de su práctica en la partería; d) respuesta ante la violencia; e) conocimiento acerca de la violencia; f) experiencias personales de violencia. Por razones de espacio, en este trabajo se abordan en profundidad las dos primeras y se retoman la d y la e.

En el cuadro I se presenta la información sociodemográfica de las parteras entrevistadas.

\section{La identificación de la violencia que sufren las mujeres durante el embarazo}

Se exploraron aspectos relacionados con la identificación de embarazadas maltratadas, tal como la intencionalidad, es decir, si las parteras realizan preguntas dirigidas a la identificación de posibles casos de violencia; si ellas toman la iniciativa o no y las razones para hacerlo.

Un aspecto recurrente en las respuestas fue que la mayoría de las entrevistadas se ha enfrentado en su 
Cuadro I

Datos generales de LAS PARTERAS EntreVistadAs. Morelos, MÉxico, 2001

\begin{tabular}{lclclc} 
& $\begin{array}{c}\text { Edad } \\
\text { (años) }\end{array}$ & $\begin{array}{c}\text { Estado } \\
\text { civil }\end{array}$ & $\begin{array}{c}\text { Número } \\
\text { de hijos }\end{array}$ & $\begin{array}{c}\text { Lugar donde } \\
\text { trabaja en M orelos }\end{array}$ & $\begin{array}{c}\text { Años } \\
\text { de práctica }\end{array}$ \\
Partera 1 & 52 & Separada & 3 & Xochitepec & 26 \\
\hline Partera 2 & 69 & Casada & 8 & Cuernavaca & 46 \\
\hline Partera 3 & 41 & Casada & 4 & Jiutepec & 15 \\
\hline Partera 4 & 54 & Viuda & 5 & Cuernavaca & 30 \\
\hline Partera 5 & 60 & Divorciada & 10 & Temixco & 35 \\
\hline Partera 6 & 52 & Casada & 4 & Cuernavaca & 25 \\
\hline Partera 7 & 57 & Viuda & 2 & Temixco & 40 \\
\hline Partera 8 & 47 & Casada & 4 & Jiutepec & 24 \\
\hline Partera 9 & 58 & Casada & 4 & Cuernavaca, & 31 \\
\hline Partera 10 & 71 & Viuda & 7 & Cuernavaca, & 54 \\
\hline Partera 11 & 54 & Casada & 3 & Atlacomulco & 40 \\
\hline Partera 12 & 76 & Viuda & 7 & Chamilpa & 57
\end{tabular}

práctica a varios casos de mujeres maltratadas; la mayoría tuvo abundantes testimonios sobre mujeres maltratadas durante el embarazo. Ante la pregunta ¿sus pacientes le reportan ser maltratadas por sus parejas?, estas fueron algunas de las respuestas:

- Sí, sí me han tocado muchas mujeres cuando están embarazadas van conmigo y me platican sus problemas (Partera 3 Ln. 582-585).

-Sí, me vienen maltratadas ya embarazadas (Partera 4 Ln. 568-569).

-Sí, hay varias experiencias muy tristes (Partera 2 Ln. 392-393).

En general, en la atención se observaron dos tendencias respecto al proceso de identificación de mujeres maltratadas. En primer lugar, aquella en donde la partera no pregunta de manera directa sobre la violencia, por una incomodidad manifiesta, y la otra se refiere a la voluntad de las mujeres para hablar, es decir, tomar la iniciativa en la narración de sus experiencias de violencia; cabe señalar que esto fue lo más común entre las parteras entrevistadas.

\section{La incomodidad para iniciar la indagación sobre la violencia}

Las parteras mostraron una abierta incomodidad para tomar la iniciativa en la indagación sobre las experiencias de violencia entre sus pacientes. Este temor de verificar algo que se sospecha, como el maltrato a una mujer embarazada, produce un sinnúmero de reacciones emocionales difíciles de manejar. El siguiente testimonio ilustra lo anterior:

- ¿Usted acostumbra preguntar sobre experiencias de violencia o maltrato en sus pacientes?

A mí no me gusta, porque bueno yo en primer lugar como que soy muy sensible, como que no acepto esa clase de maltratos, no quiero darme cuenta de eso, sé que existe pero no quiero, no lo acepto yo en mi persona, entonces yo no les pregunto. Si ellas inician la plática y quieren desahogarse tampoco yo las puedo callar y dejo que hable, que hablen todo lo que quieran, porque esa es una forma de desahogarse y pues ya si encuentro palabras como orientarlas o darles ánimo pues lo hago pero en una forma de no mezclarme en este tipo de problemas sino de buscar la forma en que ella reaccione, que recapacite y pues la ayudo. (Partera 2 Ln. 593-610).

En el testimonio anterior se expresa la intención de "ocultar" algo que se sabe que existe, que es común y afecta a un gran número de mujeres. Así, la partera reproduce una de las respuestas sociales más frecuentes ante la violencia doméstica, es decir, la negación, la cual se expresa a través del silencio alrededor de la violencia que viven las mujeres.

Además, la partera toma distancia entre ella y la mujer maltratada como una estrategia para no involucrarse, como si con ello se pudiera evitar su existencia. La reacción de la partera entrevistada se explica por la dificultad para manejar emocionalmente su reacción. Este aspecto del testimonio ilustra la necesidad de proporcionar al personal de salud las herramientas que les permitan contener sus propias emociones ante las narraciones o evidencias del maltrato en sus pacientes.

\section{La voluntad de las mujeres de hablar de la violencia}

A través de las narrativas de las parteras, se pudo apreciar las pocas limitaciones que tienen algunas mujeres para hablar de la violencia que viven, lo cual evidencia, en primer lugar, el nivel de confianza que tienen con la partera que las atiende. En segundo, la necesidad de las propias mujeres de ser escuchadas. Una partera señaló: "Ellas vienen y nos lo dicen". Los siguientes testimonios profundizan en lo anterior:

Sin preguntarles a veces empiezan a decir y a veces con eso, mire, se componen (Partera 9 Ln. 339-340).

- ¿Cómo ha identificado mujeres maltratadas en su consulta? 
- Vienen golpeadas, llorosas, llegan desveladas, muy deprimidas y pues empiezan a platicar... En otras ocasiones pues se da una cuenta porque llegan golpeadas, llorosas quejándose con uno... (Partera 12 300-308).

En otros casos, las parteras informaron llevar a cabo preguntas explícitas para identificar posibles situaciones de violencia en sus pacientes, sobre todo cuando tienen sospechas; éstas pueden ser la presencia de viejas lesiones físicas como moretones, nariz chueca, ojos morados, o por la actitud de la mujer de timidez y miedo.

Varias parteras señalaron algunas de las características que delatan a las mujeres maltratadas: la timi$\mathrm{dez}$, ser miedosas, incapaces de tomar decisiones sobre el cuidado a su salud y un descuido visible en su apariencia. Estas señales permiten preguntar de manera directa. El siguiente testimonio ilustra este aspecto:

- ¿Cómo ha identificado mujeres maltratadas en su consulta?

-Pues a veces porque son cohibidas, como que tienen miedo de verla a una de frente, como que esconden ese miedo, sienten que la va a ver uno así diferente, entonces les digo ¿te maltrata tu esposo? ¿Te golpea? ¿Pasa algo con él?

-Dime, yo te voy a ayudar, si está en mis manos yo te ayudo. Primero la confianza para que ellas puedan sacar todo lo que traigan; encima, yo también fui maltratada y no quiero que haya más mujeres así. Entonces cuando yo les empiezo a platicar algo de lo mío, así ya se sueltan (Partera 12 Ln.618-638).

Es importante observar la estrategia de la partera para identificar señales que, sabe, están relacionadas con el maltrato a la mujer; además, se pone de manifiesto su sensibilidad para dar confianza, brindar apoyo y hablar de su propia experiencia, lo cual le abre las puertas para enfrentar de manera directa la violencia que sufren las mujeres. La partera, a partir de su conocimiento empírico, pone en juego todo lo que el personal especializado ha señalado como los pasos a seguir para realizar un adecuado proceso de identificación, el cual incluye observar señales de alarma, preguntar de manera directa sobre la violencia, brindar apoyo y hacerle ver a la mujer que la violencia es algo común para muchas mujeres.

Otra partera señaló la observación de ciertos síntomas que le permiten suponer que la mujer está siendo maltratada, y entonces pregunta directamente. Este es su testimonio:

¿Cómo identifica usted a mujeres maltratadas en su consulta?
Pues a veces que le sube la presión, que hacen coraje, que traen un dolor y luego ahí les empiezo a preguntar, y me dicen: hice coraje, me peleé con mi marido, y ya de por ahí sale. Por eso se da uno cuenta, porque en el momento les sube la presión, están llorando, se sienten mal (Partera 9, Ln. 324-334).

El testimonio anterior revela cómo la experiencia de la partera le permite asociar ciertos síntomas con la violencia que sufren las mujeres, manifestando un manejo de las repercusiones de la misma en el bienestar de la embarazada. Esto se expresa mediante la presencia de un malestar generalizado que se expresa en la elevación de la presión arterial y un dolor difuso que la partera identifica claramente como consecuencia del enojo ocasionado en la mujer por su marido.

\section{Tipos de violencia que identifican las parteras}

En todos los casos, la violencia más evidente fue la de tipo físico. En varias ocasiones las parteras señalaron haber visto a sus pacientes con lesiones visibles en diferentes grados de intensidad, las cuales incluyen equimosis en cara y cuerpo, equimosis en el vientre ya avanzado el embarazo, cortaduras de machetazos en brazos y manos, labios reventados, párpados edematizados y con equimosis y costillas fracturadas.

Conviene mencionar que la violencia física identificada por las parteras varió en términos de severidad, es decir, identifican, tanto casos de violencia "leve", cuyas lesiones más frecuentes son las equimosis y contusiones en diferentes partes del cuerpo, como casos de violencia "severa", considerada así por el grado de peligrosidad de las lesiones infligidas en contra de la mujer. Los testimonios en los que se involucró formas de violencia severa fueron frecuentes en la narrativa de varias de las parteras entrevistadas.

Una de ellas narró el caso que más le ha impactado en su experiencia, y es el siguiente testimonio que se refiere a una mujer que tuvo un trabajo de parto prematuro como consecuencia de la golpiza que su marido le propinó en el tercer trimestre del embarazo:

-Me llamó una vecina que fuera a ver a la señora que se quejaba de muchos dolores, pero que ella no tenía todavía el tiempo para el parto normal. - Yo le dije- dígale que venga, aquí la voy a checar. - No- doña es que no puede caminar, la golpeó su esposo. - Estaba la pobre con el ojo cerrado, la boca bien inflamada, bien moreteada. Le dije a la señora- ¿qué le pasó?, - Es que llegó (el marido) y me pegó. Me dijo que no le servía ya como mujer, que yo nada más servía cuando no estaba embarazada.- La chequé, le dije que el bebé ya iba a nacer, salud pública de méxico / vol.46, no.1, enero-febrero de 2004 
pero yo no quería atenderla porque tenía hemorragia. Me dijo- atiéndame usted. - No- le dije -. Este parto viene mal.

La chequé y el niño no se le movía muy bien. Tenía la presión (arterial) muy alta. Yo le expliqué- usted puede tener una convulsión y que hacemos aquí. Mejor vamos, mejor yo la llevo al hospital.

-Tengo miedo- me dijo.

-No, yo la voy a acompañar.

La llevé al hospital, le expliqué al doctor: "mire a la señora la golpearon, está embarazada pero no está a término y ya tiene contracciones".

-¡Qué barbaridad! Esta señora está muy golpeada- me dijo el doctor. Le tomaron una radiografía, tenía fracturada una costilla, tenía una pierna luxada. Estaba muy mal.

La señora dijo que el marido le había dado de patadas en el vientre. Tenía el vientre todo morado.

En el hospital el bebé nació con fractura de su hombrito, le pusieron una férula y se compuso el bebé. Nació de muy bajo peso, sobre todo el bebé lloraba mucho, a pesar de comer, lloraba mucho (Partera 9 Ln. 1137-1269).

Este testimonio pone en evidencia la severidad de la violencia que algunas mujeres experimentan durante el embarazo, la cual se caracteriza por golpes y patadas dirigidas al abdomen. Otro aspecto a considerar es el nivel de control que el hombre ejerce sobre el cuerpo de la mujer, dicho deseo se ve limitado ante la presencia del otro ocupando el espacio y el cuerpo de la mujer "que le pertenece". Esta imposibilidad de uso sexual de su mujer es lo que le produce una profunda rabia y acomete directamente en contra del vientre visiblemente preñado.

El tipo de violencia como la anterior pone en evidencia el nivel de vulnerabilidad de una mujer embarazada ante un ataque de esta magnitud, tal como lo ilustran las lesiones infligidas en su contra: la fractura y la luxación. Sobre todo, la precipitación prematura del parto, la hemorragia y la pre-eclampsia, que ponen en riesgo su vida y la del bebé. El testimonio también ilustra el impacto de la violencia en el bebé en gestación como el bajo peso al nacer y las fracturas fetales.

Respecto al reconocimiento de otros tipos de violencia en la práctica de las parteras, la violencia verbal es una experiencia común entre las mujeres embarazadas. Las riñas y peleas con el marido son reportadas a las parteras por las mujeres con regularidad. En el caso de la violencia sexual, la mayoría de las parteras dejan que sean las propias mujeres quienes hablen de este tipo de experiencias pues lo consideran muy delicado para abordarlo de manera directa. Algunas de ellas comentaron que sus pacientes les in- forman que los maridos las acosan sexualmente durante el embarazo o en el puerperio.

Otra experiencia común en las parteras fue la identificación de casos de mujeres violadas, tanto las que son abusadas por sus parejas como aquellas que son violadas por desconocidos $\mathrm{y}$, como consecuencia, la mujer queda embarazada.

\section{Discusión}

Es importante resaltar algunos de los hallazgos más sobresalientes del estudio. En primer lugar, la familiaridad de las parteras con la violencia que viven de manera cotidiana las mujeres embarazadas, lo cual arroja luz sobre las circunstancias y condiciones en las que se presenta la violencia hacia las mujeres embarazadas, y cuyos primeros datos generales habían sido reportados por los estudios de prevalencia. $17, *$

Además, es importante hacer una comparación entre la experiencia de las parteras y lo comunicado por los médicos del primer nivel de atención del municipio de Cuernavaca en un estudio anterior, ${ }^{19}$ en dicho estudio los médicos señalaron que en su opinión la violencia durante el embarazo era una situación poco frecuente en la población que atienden, pues sus experiencias en la identificación de mujeres embarazadas maltratadas eran escasas. Esta discrepancia entre ambos pone de manifiesto diferencias sustanciales entre ambas prácticas; por ejemplo, los distintos conceptos de atención a la salud, el tipo de interacción entre quién atiende y quién consulta, la definición de su campo de intervención, por mencionar las más importantes. En el caso de las parteras ellas están insertas en el mismo contexto cultural y geográfico de las mujeres que atienden, su relación está definida más horizontalmente y, por lo tanto, existe menos distancia entre una y otra. Esta es una diferencia sustancial en comparación con la relación médico-paciente en el escenario institucional. ${ }^{\ddagger}$

Las parteras, si bien no preguntan rutinariamente de manera directa, sí responden ante la confesión de las mujeres que sufren violencia, ya sea: a) a través de una escucha atenta que les permite a las mujeres hablar hasta que quieran de su problema,

\footnotetext{
* Castro R, Valdez R, Arenas L, Ruiz A. Violencia contra mujeres embarazadas en Morelos (México): un estudio sociológico sobre prevalencia y severidad. 2001. En prensa.

‡ Para profundizar en este aspecto se puede consultar Cao Laura. Entre parteras y médicos. Diálogos sobre atención a la salud. Asociación Mexicana de Población. México, en Los silencios de la salud reproductiva: violencia, sexualidad y derechos reproductivos. Fundación MacArthur/ Asociación Mexicana de Población, México, 1998: 193-222.
} 
proporcionando algún consejo de qué hacer; $b$ ) atendiendo su malestar físico o emocional a través de diferentes intervenciones como masajes o sobadas, infusiones para los nervios, y c) como acompañantes a diferentes instancias para que reciban atención donde pueden pedir apoyo para resolver su problema de violencia. ${ }^{*}$ Esta respuesta de las parteras es consistente con lo reportado por González (1998) en una comunidad indígena del estado de Puebla. ${ }^{18}$

Otro aspecto a resaltar desde la experiencia de las parteras se refiere a las señales no físicas de la violencia que algunas identificaron en las mujeres maltratadas, como la timidez, ser miedosas, incapaces de tomar decisiones sobre el cuidado a su salud y un descuido visible en su apariencia. Estas características también fueron reportadas por algunos médicos del primer nivel de atención; sin embargo, es importante profundizar en la asociación de la violencia doméstica con el descuido de la salud de las mujeres. Si bien en la mayoría de los estudios sobre violencia y salud se resalta el impacto de la violencia en términos de lesiones físicas, una forma de impacto poco reconocida, pero con serias consecuencias, se refiere a la imposibilidad de las mujeres maltratadas de tomar decisiones sobre el cuidado para su salud. Este dato es de suma importancia para el personal de salud, tanto médicos como parteras, que atienden a mujeres maltratadas embarazadas, ya que debe ser tomado en cuenta para la identificación de la violencia y no ser malinterpretado como un simple descuido. Por otro lado, esta reacción de la mujer maltratada refleja el impacto de la violencia en su psique y su cuerpo, hecho que ha sido documentado en la literatura especializada, la cual señala las alteraciones cognitivas como consecuencia de la violencia, entre las que resalta la pérdida de noción del peligro en la cual se encuentran. ${ }^{24}$

Para finalizar, llama la atención la abundancia de testimonios de mujeres que fueron golpeadas en el abdomen en un estado avanzado del embarazo. Este aspecto ha sido reportado, tanto en el ámbito nacional como internacional. En México, en 1990, en un estudio a población abierta, se reportó que $20 \%$ de las mujeres maltratadas había experimentado golpes en el vientre estando embarazadas. ${ }^{25}$ En el ámbito internacional Bohn, en 1990, ha señalado que la violencia es más frecuente y severa durante el embarazo, y con mayor frecuencia dirigida al abdomen. ${ }^{26}$

Con base en lo anterior proponemos las siguientes recomendaciones a partir de los resultados del

\footnotetext{
* Este aspecto se aborda en otro documento derivado de la misma investigación.
}

estudio. En primer lugar, es urgente incluir a las parteras dentro de los planes y programas para atender a la violencia familiar desde el sector salud, debido a que la práctica de las parteras va más allá de atender a las embarazadas.

Por otro lado, la mayoría de las parteras entrevistadas expresó interés en conocer más sobre violencia, sus repercusiones en la salud y, sobre todo, cómo poder ayudar a las mujeres que demandan su apoyo y solicitan sus consejos. En este sentido, se propone diseñar un manual y un programa de capacitación sobre la materia, especialmente diseñado para las parteras, tal como se hizo en el caso del personal de salud oficial. $^{27}$

\section{Agradecimientos}

En primer lugar, un especial agradecimiento a las parteras que amablemente decidieron participar en el estudio. Su disposición fue una vez más evidente en el momento de la entrevista. A la maestra Margarita Avilés (INAH- Morelos) por haber facilitado el contacto con las que conforman el grupo de parteras del Jardín Etnobotánico con el cual lleva trabajando más de 10 años. A la maestra Laura Badillo, por su apoyo en la realización de algunas entrevistas. A Elsa Avilés, quien apoyó en la trascripción de las mismas. Finalmente, al Programa Interdisciplinario de Estudios de la Mujer, del Colegio de México, por su confianza y apoyo; sin este respaldo el trabajo no hubiera sido posible.

\section{Referencias}

1.Agostoni C. Médicos y parteras en la ciudad de México durante el porfiriato. En: $C$ ano $G$, Valenzuela $G$ J, coords. C uatro estudios de género en el México urbano del siglo XIX. México,DF: PUEG -Porrúa, 2001:7195.

2. Dávalos M. El ocaso de las parteras. Cuicuilco Revista de la Escuela N acional de Antropología e Historia 1996;2(6):197-200.

3. Q uezada N . Enfermedad y maleficio. 2da edición. México, DF: Universidad N acional Autónoma de México /Instituto de Investigaciones Antropológicas, 2000.

4. Menéndez E. Modelo hegemónico, modelo alternativo subordinado, modelo de autoatención. C aracteres estructurales. En: C ampos N avarro R, comp. La antropología médica en México. México: Instituto Moral Universidad Autónoma Metropolitana, 1992:97-114.

5. Campos-N avarro R. Prácticas médicas populares: algunas experiencias sobre el proceso de autoatención curativa. En: C ampos- $N$ avarro R, comp. La antropología médica en México. México, DF: Instituto Moral Universidad Autónoma Metropolitana,1992:186-210.

6. Cao-Romero L. Papel de la partería para una maternidad sin riesgos en México: ¿tradición o profesión? En: Elú MC, Santos- Pruneda E, ed. Una nueva mirada a la mortalidad materna en México. México: Fondo 
de Población de las N aciones Unidas/ Population Council, 1999,vol 1:195-205.

7. Cosminsky S. La atención del parto y la antropología médica. En: Campos $N$ avarro R, comp. La antropología médica en México. México, DF: Instituto Mora/ Universidad Autónoma Metropolitana, 1992:vol II 139-160.

8. 0 rtega-C anto J, Hoil-Santos J, Lendechy-G rajales A. Proceso reproductivo femenino: género, representaciones y actores sociales. Una reflexión desde el contexto yucateco. En:Tuñón PE, coord. Género y salud en el sureste de México. México, DF: EC O SUR/C onsejo Estatal de Población de Chiapas, 1999;vol 2: 424-445.

9. C astañeda X. Embarazo, parto y puerperio: conceptos y prácticas de las parteras en el estado de Morelos. Salud Publica Mex 1992;34:

528-532.

10. Instituto Mexicano del Seguro Social. Encuesta de Programas IMSSCOMPLAMAR y la Unidad de Investigación en MedicinaTradicional y D esarrollo de Medicamentos del IMSS. México, DF: Instituto Mexicano del Seguro Social, 1984.

11. Secretaría de Salud. Encuesta N acional sobre Fecundidad y Salud (Enfes), 1987. México, D F: Secretaría de Salud, 1989.

12. G azmararian JA, Lazorick S, Spitz AM, Ballard TJ, Saltzman LE, Marks

$J S$. Prevalence of violence against pregnant women. JAMA 1996;24(275):1915-1920

13. Bullock L, McFarlane J.The birth weight battering connection. Am J N urs 1989;89(9):1153-1155.

14. Pearlman CA,T intinalli J, Lorenz R. Blunt trauma during pregnancy. N Engl J Med 1990; vol 323 (23):1609-1613.

15. Berenson $A B, W$ iemann $C M$, W ilkinson $G S$, Jones W A, Anderson GD. Perinatal morbidity associated with violence experienced by pregnant women. Am J 0 bstet Gynecol 1994;170(6):1760-1766.

16. Moreno RA. Mujer, embarazo y violencia. Síndrome de abuso en mujeres embarazadas. Rev Colombiana 0 bstet Ginecol 1996;47(2): 87-91.

17.Valdez R, Sanín LH. La violencia doméstica durante el embarazo y su relación con el peso al nacer. Salud Publica Mex 1996;38(5):1-11.

18. González $\mathrm{S}$. La violencia doméstica y sus repercusiones en la salud reproductiva en una zona indígena (C uetzalan, Puebla).En: Los silencios de la salud reproductiva: violencia, sexualidad y derechos reproductivos.
México, DF. Fundación McArthur / Asociación Mexicana de Población, , 1998:17-54.

19.Valdez R. Respuesta del personal médico en la atención de mujeres maltratadas durante el embarazo: el caso de la consulta prenatal en los Centros de Salud de Cuernavaca, Morelos. En:Torres M, ed.Violencia contra las mujeres en diferentes contextos culturales. México, DF: El Colegio de México, 2003.

20.Arcos E, Molina I, Repossi A, U arac M, Ritter P,Arias L. Prevalencia y perfil de violencia doméstica en mujeres embarazadas. Rev Mujer Salud 2000;2:4-11.

21. Secretaría de Salud, N orma 0 ficial Mexicana para la prestación de servicios de salud. Criterios para la atención médica de la violencia familiar. (N O M-190-SSA 1-1999), México DF: Diario 0 ficial de la Federación 8 de marzo, 2000.

22.A vilés M. Medicina tradicional: plantas empleadas por parteras empíricas del estado de Morelos (Tesis de licenciatura en biología). Cuernavaca, Morelos: Universidad Autónoma del Estado de Morelos, 1985.

23. Marcos S,A vilés M. Entre la medicina y la tradición: las parteras de Morelos. En: Comité Promotor por una Maternidad sin Riesgos en México. Maternidad sin riesgos en Morelos. México, DF: Comité Promotor por una Maternidad sin Riesgos en México, 1996:115-129. 24.Valdez R, Juárez C. Impacto de la violencia doméstica en la salud mental de las mujeres: análisis y perspectivas en México. Salud mental 1998;21(6):1-10

25.Valdez R, Shrader E. Características y análisis de la violencia doméstica en México: el caso de una microrregión de Ciudad $\mathrm{N}$ etzahualcóyotl. En:A un la luna a veces tiene miedo... México, D F: Centro de Investigación y Lucha contra laViolencia Doméstica, 1992:35-49

26. Bohn DK. Domestic violence and pregnancy. Implications for practice. J N urs Midwifery 1990;35(2):86-98.

27. Elú MC, Santos E,Valdez R,Arenas L, C astro R, Rivera M et al. Carpeta de apoyo para la atención en los servicios de salud de mujeres embarazadas víctimas de violencia. México, DF: Comité Promotor por una Maternidad sin Riesgos en México, 2000:34. 